\title{
Effectiveness of Hall Technique for Primary Carious Molars: A Systematic Review and Meta-analysis
}

\author{
Sheikh Bilal Badar ${ }^{1}$, Sadia Tabassum², Farhan Raza Khan ${ }^{3}$, Robia Ghafoor ${ }^{4}$
}

\begin{abstract}
Aim: The objective of the present systematic review was to assess the outcomes of Hall technique (HT) on primary carious molars and compared it with the conventional dental restorations.

Materials and methods: The systematic review was registered with Prospero registry (CRD42015020445) to answer the following research question: Is HT a better restorative option compared to other techniques for restoration of carious primary molars? In addition to exploring various health sciences databases, hand search was also done using following key terms in different permutations: (Hall technique OR Hall's technique OR preformed metal crown OR stainless steel crown) AND (caries OR carious molar OR deciduous tooth OR baby tooth OR milk tooth OR primary tooth). The outcome of interest was success of the restoration achieved with either method.

Results: Five studies were included (two RCTs, one quasi-experimental trial, and two retrospective). A total of 1775 teeth were assessed, of which 1325 teeth were restored using HT. The retrospective studies showed no difference between HT and other methods whereas the RCTs and quasi-experimental favored $\mathrm{HT}$ over other treatment modalities. Meta-analysis significantly favored HT over conventional restorations [risk ratio $5.55(3.31-9.30)]$ ( $p$ value $\leq 0.001)$.
\end{abstract}

Conclusion: HT appeared demonstrated higher success and significantly outperformed the conventional restorations.

Keywords: Deciduous carious molars, Hall technique, Metal crowns, Preformed metal crowns.

International Journal of Clinical Pediatric Dentistry (2019): 10.5005/jp-journals-10005-1666

\section{INTRODUCTION}

According to the WHO report in 2003, dental caries affects around $60-90 \%$ of children worldwide. ${ }^{1}$ Not only the developing countries but the children residing in developed countries are affected with caries. $^{2,3}$ In primary dentition, the most commonly affected teeth are the primary molars. ${ }^{4}$ If left unmanaged, dental caries progresses to involve pulp leading to pain and infection, the consequences of which are unnecessary suffering that cannot be contained with pharmacological means. ${ }^{5}$ Despite the knowledge on prevention, a big proportion of caries in pediatric patients is unfortunately left untreated. ${ }^{6,7}$ The lack of care is attributed to problems such as inaccessible dental care, lack of affordability, inadequate dentist training, unrealistic patient's expectation, child's inability to cooperate and the desire of not to frighten the child by refraining away from the required intervention. ${ }^{7-9}$ Untreated caries can have a social bearing on the child's quality of life as well as economic consequences on the parents. Therefore, timely management of carious primary molars is essential to prevent premature tooth loss, to maintain physiological space for the developing permanent molars, and to help in speech and mastication, thus enhancing self-confidence and provide improved quality of life of the pediatric patients. ${ }^{10}$

Conventionally, a cavitated carious lesion is managed with a directly placed dental restoration. A variety of restorative materials including dental amalgams, composite resins, compomers, and resin-modified glass ionomer have been used to treat carious primary teeth. ${ }^{11-13}$ However, despite the advancement in the restorative dentistry and dental material sciences, tooth preparation is usually needed to place a restoration. Tooth preparation in deeper lesions require need of local anesthetics, has the potential to expose dental pulp, and can lead to a structurally weakened tooth structure. Sometimes, the primary tooth is already too
${ }^{1}$ Department of Surgery, Aga Khan University, Karachi, Pakistan

${ }^{2}$ Dental Department, Jinnah Medical Dental College, Karachi, Pakistan

${ }^{3}$ Department of Surgery, Dental Section, Aga Khan University, Karachi, Pakistan

${ }^{4}$ Department of Dentistry, Aga Khan University Hospital, Karachi, Pakistan

Corresponding Author: Sadia Tabassum, Dental Department, Jinnah Medical Dental College, Karachi, Pakistan, Phone: +92 2134931886, e-mail: sadyatabassum@gmail.com

How to cite this article: Badar SB, Tabassum S, Khan FR, et al. Effectiveness of Hall Technique for Primary Carious Molars: A Systematic Review and Meta-analysis. Int J Clin Pediatr Dent 2019;12(5):445-452.

Source of support: This research did not receive any specific grant from funding agencies in the public, commercial, or not-for-profit sectors

Conflict of interest: None

compromised (particularly in cases of multi-surface decay) at the presentation, making the restorative procedure challenging and that too in a patient that is young and mostly apprehensive. ${ }^{14-16}$

Preformed metal crowns have shown significant clinical success and are considered as a favorable restorative option for moderate to severe caries involving two or more surfaces of primary molars., ${ }^{4,16}$

The durability of preformed metal crowns is its fundamental advantage over a multi-surface directly placed restoration with a documented 5 -years survival rate reaching up to $100 \% .^{17}$ The preformed metal crowns are less technique sensitive and offers coronal coverage to the affected tooth. ${ }^{18}$ Despite being gold standard treatment modality and being favored by the specialists,

() The Author(s). 20190pen Access This article is distributed under the terms of the Creative Commons Attribution 4.0 International License (https://creativecommons. org/licenses/by-nc/4.0/), which permits unrestricted use, distribution, and non-commercial reproduction in any medium, provided you give appropriate credit to the original author(s) and the source, provide a link to the Creative Commons license, and indicate if changes were made. The Creative Commons Public Domain Dedication waiver (http://creativecommons.org/publicdomain/zero/1.0/) applies to the data made available in this article, unless otherwise stated. 
preformed metal crowns have not yet become the part of routine treatment in UK. ${ }^{19-21}$

The placement of stainless steel crown is sometimes challenging as it requires patient cooperation, which is difficult to achieve in pediatric patients. For the purpose of simplifying the procedure and making it receptive to the patients, Dr Hall devised a technique of stainless steel crown placement in children that does not require local anesthesia, or caries removal or any sort of tooth preparation. ${ }^{22}$ This technique is based on the scientific evidence that caries progression gets arrested once an effective marginal seal is achieved..$^{23} \mathrm{~A}$ properly placed stainless steel crown denies the cariogenic bacteria of an environment that is conducive for acidic demineralization of the inorganic and proteolytic disintegration of the organic component of the tooth structure. Randomized controlled trials performed to assess the effectiveness of Hall technique (HT) have shown favorable results. ${ }^{24-27}$

Innes et al. ${ }^{28}$ did a systematic review on the effectiveness of preformed crowns on carious primary molar teeth but were unable to draw any inference on the success and longevity of crown placed with conventional technique compared to those placed with HT. Thus, there is a lack of highest level of evidence regarding the superiority or noninferiority of the either of the techniques in the management of carious primary molars. The present systematic review is, therefore, planned to explore the effectiveness of HT in the management of carious primary molars.

\section{Materials and Methods}

The systematic review was carried out using the PRISMA guidelines. ${ }^{29}$ The focused question according to PICOS framework was following:

Population: Children with asymptomatic carious primary molar teeth.

Intervention: Placement of a crown according to the Hall technique.

Comparison: Caries removal followed by standard control restorations or stainless steel crown.

Outcome: Retention of deciduous tooth and/or absence of pulpal symptoms.

Studies: Randomized controlled trials, quasi-experimental studies, cohort, and retrospective studies.

\section{Search Strategy}

Following the registration of the systematic review protocol with PROSPERO (Reference \# CRD42015020445), an advanced search was carried out in five major databases including PubMed, CINAHL plus, Cochrane library, Dentistry and Oral Sciences, and Scopus. The MeSH keywords used for the search included ("Hall technique" OR "hall's technique" OR "preformed metal crown" OR "preformed stainless steel crown" OR "steel crown") AND ("caries" OR "carious molar" OR "deciduous tooth OR "baby tooth" OR "milk tooth" OR "primary tooth." In all the databases, the filter was included for articles in English language carried out in both genders of human population. In addition to this, hand search was performed using the mentioned keywords in clinical trial registries (clinicaltrials.gov and BMC trial registry), IADR and Cochrane databases and Google Scholar for the recent studies. Furthermore, in the search of grey literature, SIGLE (opengrey.eu) and greylit.org databases were explored which also contained articles and theses in languages other than English. For the registered protocols mentioned in the trial registries, authors were contacted regarding the estimated time remaining for the results.

\section{Selection Criteria}

Inclusion criteria for the selected studies include English language research articles with full-text manuscript. In addition to that all clinical trial, randomized clinical trials, case control and cohort studies on the relevant topic were included, whereas case reports, case series, narrative reviews, single-arm longitudinal studies, and in vitro studies were excluded. The abstract without full-text articles were also excluded from conducting and reporting of the present systematic review. Furthermore, only those comparative studies were included which had a minimum follow-up period of three months. The PRISMA flow diagram is shown in Flowchart 1.

\section{Data Extraction}

Articles retrieved after the literature search were reviewed by the study investigators to exclude studies that were duplicated or irrelevant. Further screening of the remaining articles was performed by two reviewers independently, to assess the eligibility of the potential articles to be included for the systematic review. In case of any disagreement at this stage, the third author was consulted. From the finally selected articles, the relevant information was retrieved including participant age, teeth treated, type of intervention, follow-up period, longevity of restoration, success or failure, and presence of any clinical or radiographic signs of pulpal pathology on a customized proforma. Furthermore, the studies having quantitative data were subjected to meta-analysis to compute a summary effect on the risk ratio. Random effect model was adopted as it was assumed that the trials have variability in the conduct and reporting of outcomes. Heterogeneity was determined with $P^{2}$ statistics.

\section{Risk of Bias Assessment}

The guidelines of the Cochrane Collaboration's Tool for assessing risk of bias in randomized trials ${ }^{30}$ were followed for the selected clinical trials. This included the evaluation of random sequence generation, allocation concealment, blinding of participants, blinding of outcome data, selective reporting, and other biases in the included clinical trials. The assessments of these parameters were marked as of high, low, or unclear risk. The risk of bias for the retrospective studies was also evaluated using the Agency for Healthcare Research and Quality (AHRQ) criteria (as shown in Annexure). ${ }^{31}$

These assessments were carried out by two authors with discussion and mutual agreement. In case of any disagreements or discrepancies, the opinion of the third reviewers was sought and that was considered final.

\section{Results \\ Description of Studies}

A total of 900 articles and abstracts were retrieved (Flowchart 1). After initial exclusion of studies, ten studies were found to be relevant to the research question. Studies conducted by Innes et al. ${ }^{27}$ and Santamaria et al. ${ }^{24,25}$ were found to be published with different follow-ups; therefore, studies ${ }^{24,25,27}$ with less follow-ups were excluded to control the duplication of studied data. Trial protocols by Tonmukayakul et al. ${ }^{32}$ and Hesse et al. ${ }^{33}$ were also excluded on account of nonavailability of the study results as they were expected to be published later. While searching for grey literature in SIGLE, out 
Flowchart 1: PRISMA flow diagram
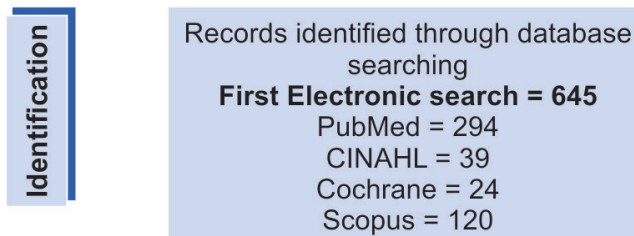
searching

First Electronic search $=645$

PubMed $=294$

CINAHL $=39$

Cochrane $=24$

Scopus $=120$

Dent \& Oral Sci. $=168$
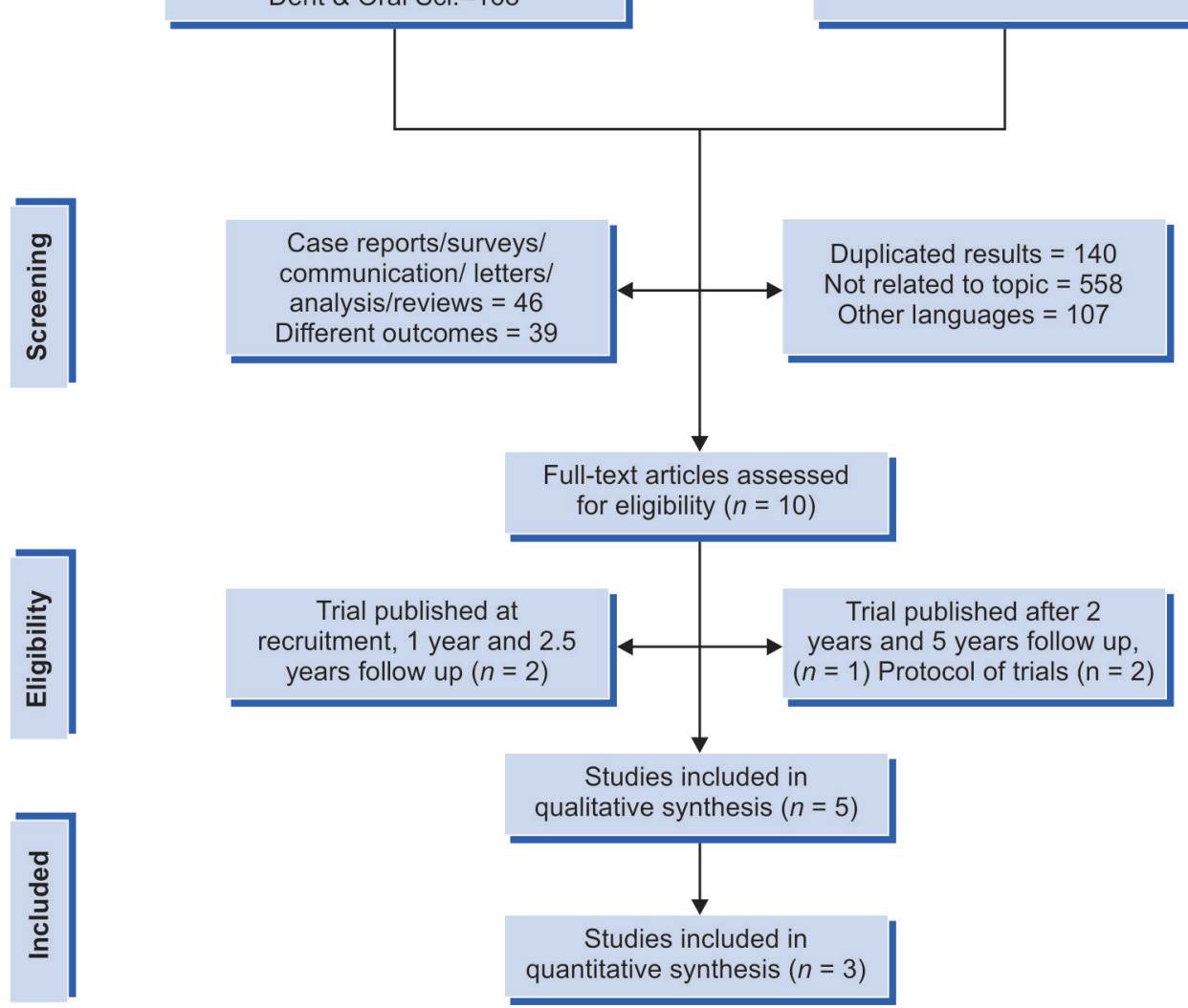
through other sources Additional search $=\mathbf{2 5 5}$

Clinical trial registry $=7$

BMC trial registry $=107$

SIGLE $=72$

Hand search $=69$

\begin{tabular}{|c|c|c|c|c|c|}
\hline \multirow{3}{*}{ Study or subgroup } & \multicolumn{2}{|c|}{ Conventional restoration } & \multicolumn{3}{|c|}{ Favors [Hall technique] } \\
\hline & Events & Total & Events & Tota & Weight \\
\hline & 20 & 63 & 5 & 84 & $31.3 \%$ \\
\hline Innes et al. 2018 & 44 & 91 & 7 & 91 & $48.4 \%$ \\
\hline Santamaria et al. 2017 & 19 & 58 & 3 & 40 & $20.2 \%$ \\
\hline Total $(95 \% \mathrm{Cl})$ & & 212 & & 21 & $100.0 \%$ \\
\hline $\begin{array}{l}\text { Total events } \\
\text { Heterogeneity: } \text { Tau }^{2}=0 . \\
\text { Test for overall effect: } Z\end{array}$ & $\begin{array}{c}83 \\
0.00 ; \mathrm{Chi}^{2}=0 \\
\mathrm{Z}=6.49 \cdot(\mathrm{P}\end{array}$ & $=2(P=$ & $\begin{array}{c}15 \\
87 ; P=0 \%\end{array}$ & & \\
\hline
\end{tabular}

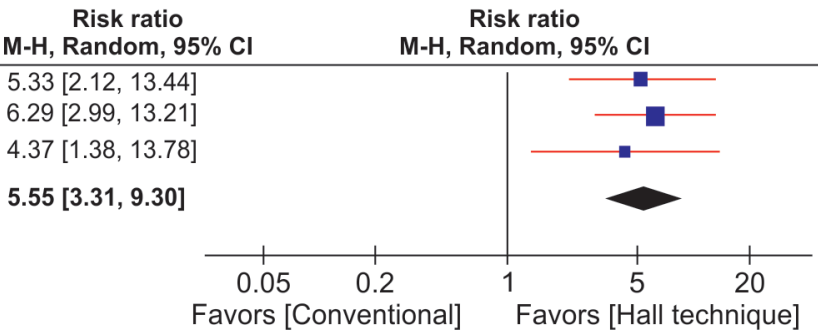

Fig. 1: Forest plot showing success of Hall technique vs conventional restoration

of 72 studies only one doctoral thesis was found to be relevant. ${ }^{34}$ It was in French language which was translated to English; however, it was excluded due to lack of any intervention provided by the investigators. Finally, only five studies ${ }^{26,35-38}$ were evaluated in the present systematic review. After data extraction and critical analysis, three studies ${ }^{26,37,38}$ with numeric data on the success of HT were subjected to the meta-analysis (Fig. 1).

\section{General Characteristics of Included Studies}

Out of five studies, the total number of teeth assessed were 1775 (Table 1). Of these, 1325 teeth were restored using HT and the rest were restored with other techniques including conventional restorations or nonrestorative care. The three clinical trials had 280 teeth managed with HT whereas 1,045 teeth were restored with the same in the two retrospective studies. The follow-up period for the evaluation of teeth restored using $\mathrm{HT}$ varied from 15 months to 5 years.

Table 2 shows the outcome described in the included studies. Innes et al. ${ }^{35}$ conducted a retrospective analysis of 978 preformed metal crowns which were followed for 5 years. This study showed that the preformed metal crowns cemented using HT showed $67.6 \%$ survival at 5 years follow-up. Ludwig et al. ${ }^{36}$ performed a chart review of already placed crowns using HT that were followed-up till 37 months and reported $97 \%$ success rate of the treatment, as 

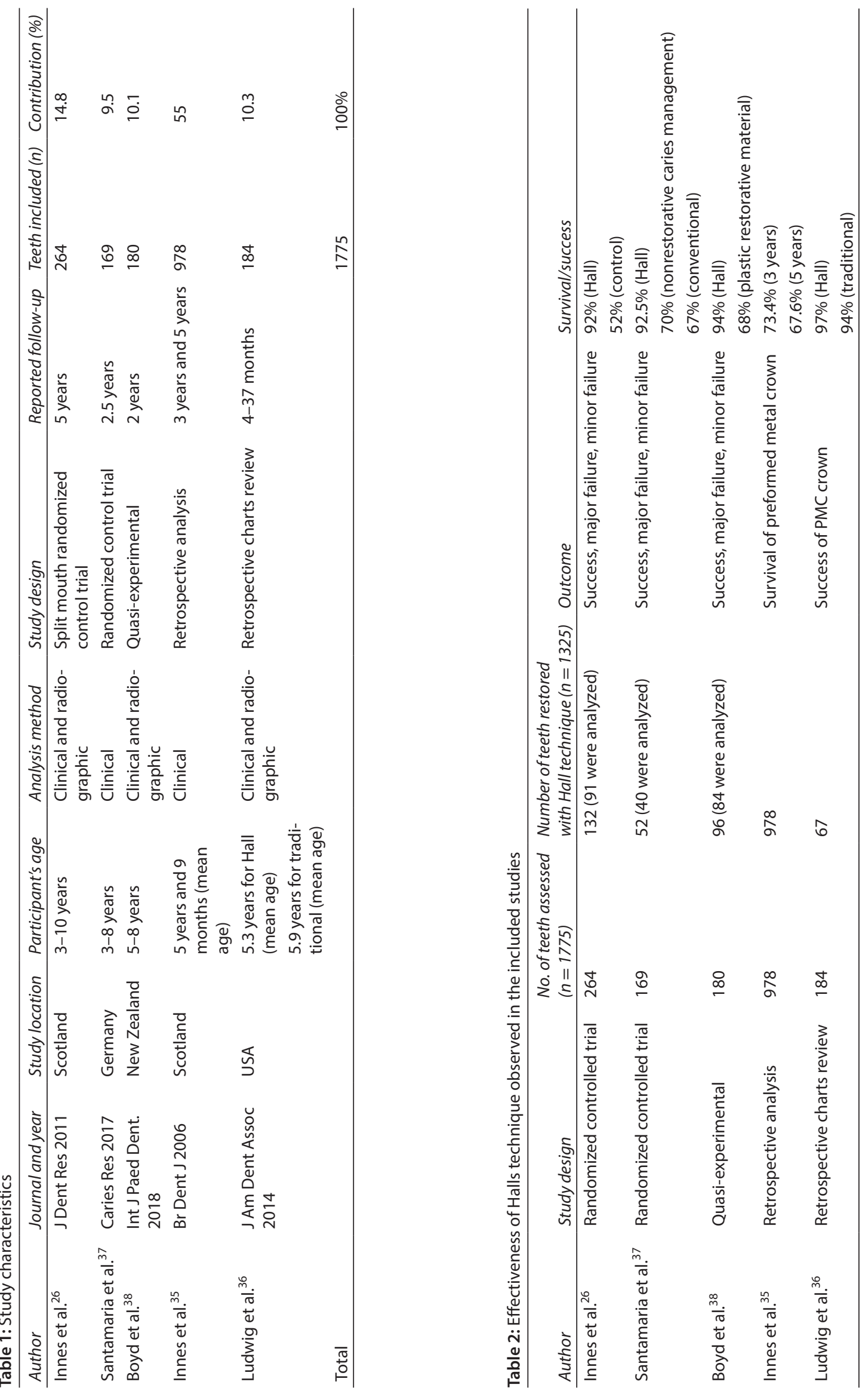


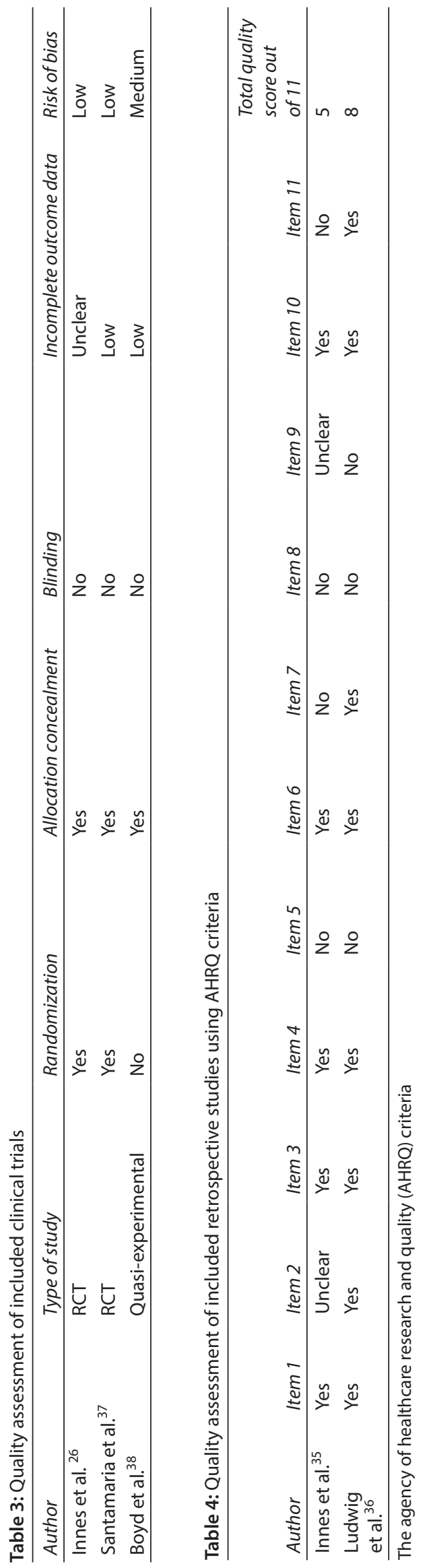

opposed to the $94 \%$ of ascribed success of crowns cemented with traditional technique.

Innes et al. ${ }^{26}$ carried out a split mouth randomized controlled clinical trial comparing HT with other restorative options. After 5 years of follow-up and evaluation of major and minor failures, it was evident that HT outperformed the conventional restorations. HT had only $3 \%$ failures compared to $16.5 \%$ failure in the conventional restorations. Santamaria et al. ${ }^{37}$ compared HT with the conventional treatment (and as well as nonrestorative treatment). This study had 2.5 years follow-up, and their results further consolidate the evidence in favor of HT.

\section{Risk of Bias}

Quality assessment of the included studies is shown in Tables 3 and 4 . Being randomized controlled trials, Innes et al. ${ }^{26}$ and Santamaria et al. ${ }^{37}$ showed low risk of bias. However, Boyd et al.'s study, ${ }^{38}$ which is a quasi-experimental study, lacked randomization of the participants and therefore showed medium risk of bias. Owing to the nature of intervention, blinding of the participant and care providers was not possible in the trials. For retrospective studies, ${ }^{35,36}$ the AHRQ criteria ${ }^{31}$ were adopted, the result of which is shown in Table 4.

\section{Discussion}

The objective of the present systematic review was to evaluate the effectiveness of HT in the management of carious primary teeth. The stringent inclusion criteria allowed the scrutiny of the studies; thus, only five studies were finally evaluated to get an unbiased answer to the research question.

Of the included studies, three compared stainless steel crown placed with $\mathrm{HT}$ with various control restorations. ${ }^{26,36,37}$ Innes et al. ${ }^{26}$ conducted a split mouth randomized control trial in which they compared stainless steel crowns placed with HT with the control restoration in the contra lateral tooth. This control restoration was determined to be any restoration placed by the clinician. Santamaria et al. ${ }^{37}$ compared the success of HT with nonrestorative caries treatment and conventional restorations. Ludwig et al. ${ }^{36}$ in their retrospective study compared crowns placed with HT and crown placed after conventional preparation of the tooth structure. Innes et al. ${ }^{35}$ conducted a retrospective analysis too where they evaluated Hall technique without any comparator. These studies inferred HT as a successful treatment modality for the management of carious deciduous molars. This technique is found to be equally effective compared to alternative options which are aggressive and require cooperation from the pediatric patient.

The authors of the included studies ${ }^{26,35-38}$ followed cases up to 5 years (range 4 months to 5 years) and evaluated the success or survival of crowns cemented with HT. The criterion for success was the presence of crown on the affected tooth or tooth exfoliated without any signs or symptoms that required any further intervention. In contrast to this, teeth that showed signs of irreversible pulpitis or dental abscess, inter radicular radiolucency, restoration loss, or tooth became nonrestorable were considered as failure. ${ }^{26,35-38}$

HT exhibited considerably higher success or survival rate as opposed to other directly placed restorations. ${ }^{24,26,37}$ While comparing preformed crowns placed with traditional technique (involving removal of the carious dentine under local anesthesia) with $\mathrm{HT}$, it was found that $\mathrm{HT}$ had a comparable or even higher success rate. ${ }^{36}$ It can, therefore, be speculated that placement of 
stainless steel crown is more predictable treatment option whether placed with or without HT for the management of carious primary molars. Innes et al. ${ }^{35}$ also documented high survival rate for crowns placed with HT. However, due to the retrospective design and lack of comparison group in the two studies, ${ }^{35,36}$ the conclusions were not robust.

The limitation of the present systematic review is that it is unable to evaluate the attitude of patients and clinicians over HT. Only Santamaria et al. ${ }^{25}$ in the preliminary report of the trial evaluated the children behavior, pain perception during treatment, and dentist opinion regarding treatment. They concluded that HT and nonrestorative technique were shown to be better tolerated by children as opposed to conventional restorative management. However, the level of comfort described by children was same for all the groups. Furthermore, a trial by Tonmulayakul et al. ${ }^{32}$ is currently underway. That trial is likely to determine the clinical outcomes including the cost effectiveness of the stainless steel crowns placed on the carious primary molars, thus would generate more information on HT. A trial by Hesse et al. ${ }^{33}$ is also in progress which is aimed at the outcomes of Atraumatic Restorative Technique and HT in the scenario of limited resources and technically challenging situations.

The meta-analysis done over three trials on the comparison of HT vs conventional methods of restoring primary carious teeth showed that HT is far more successful than the comparative treatment modalities 5.55 (3.31-9.30; $p$ value $<0.001$ ).

HT is in the profession for more than a decade with its effectiveness proven with a number of studies, and its practice is still limited to pediatric dentists. ${ }^{23}$ General dentists have still not internalized the HT technique and it appears that they are hesitant in adopting this approach. A probable reason might be the fact that it is in conflict with teaching operative dentistry in schools where mechanical debridement is the essential prerequisite of any restoration. ${ }^{23}$ However, due to improved understanding of cariology, and high-quality research evidence in the form of systematic review and meta-analysis, it can be speculated that in the future, HT will be not only be incorporated in the curriculum of dental schools but will be practiced by all dentists who offer restorative care to children. This will help in saving lot of financial resources and avoiding unnecessary use of sedation and general anesthesia in children. ${ }^{9}$

\section{Conclusion}

Within the limitation of the present systematic review, it can be concluded that Hall technique is a not only a predictable restorative option but it has significantly outperformed the conventional method of treatment of carious primary molars. The success rate of Hall technique is 5 times that of the conventional restorative techniques.

\section{Authors' Contribution}

All authors contributed significantly. Sheikh Bilal Badar and Sadia Tabassum conceived the idea, searched the literature, and selected the studies. Sheikh Bilal Badar and Sadia Tabassum composed the manuscript. Farhan Raza Khan interpreted the data and critically reviewed the article. Robia Ghafoor helped in the discussion and reviewed the manuscript.

\section{ACKNOWLedgment}

The authors would like to acknowledge Khawaja Mustafa, Head librarian, Aga Khan University for helping in the literature search.

\section{References}

1. Petersen $P E$, Bourgeois $D$, et al. The global burden of oral diseases and risks to oral health. Bull World Health Organ 2005;83:661-669. DOI: S0042-96862005000900011.

2. Colak H, Dulgergil CT, et al. Early childhood caries update: a review of causes, diagnoses, and treatments. J Nat Sci Biol Med 2013;4:29-38. DOI: 10.4103/0976-9668.107257.

3. Dye BA, Mitnik GL, et al. Trends in dental caries in children and adolescents according to poverty status in the United States from 1999 through 2004 and from 2011 through 2014. J Am Dent Assoc 2017;148(8):550-565. DOI: 10.1016/j.adaj.2017.04.013.

4. Innes NP, Ricketts DN, et al. Preformed metal crowns for decayed primary molar teeth. Cochrane Database Syst Rev 2007; CD005512. DOI: 10.1002/14651858.CD005512.pub2.

5. Vargas CM, Crall JJ, et al. Sociodemographic distribution of pediatric dental caries: NHANES III, 1988-1994. J Am Dent Assoc 1998;129: 1229-1238. DOI: 10.14219/jada.archive.1998.0420.

6. Levine RS, Pitts NB, et al. The fate of 1,587 unrestored carious deciduous teeth: a retrospective general dental practice based study from northern England. Br Dent J 2002;193:99-103. DOI: 10.1038/ sj.bdj.4801495.

7. Garg S, Rubin T, et al. How willing are dentists to treat young children?: a survey of dentists affiliated with Medicaid managed care in New York City, 2010. J Am Dent Assoc 2013;144:416-425. DOI: 10.14219/ jada.archive.2013.0135.

8. Milsom K, Tickle M, et al. Does the dental profession know how to care for the primary dentition? Br Dent J 2003;195:301-303. DOI: 10.1038/ sj.bdj. 4810525.

9. Hyde AC, Rogers HJ, et al. An Overview of Preformed Metal Crowns. Part 2: The Hall Technique. Dent Update 2015;42(939-942):44. DOI: 10.12968/denu.2015.42.10.939.

10. Rogers HJ, Batley HA, et al. An Overview of Preformed Metal Crowns. Part 1: Conventional Technique. Dent Update 2015;42(933-936):8. DOI: $10.12968 /$ denu.2015.42.10.933.

11. Kilpatrick NM, Neumann A. Durability of amalgam in the restoration of class II cavities in primary molars: a systematic review of the literature. Eur Arch Paediatr Dent 2007;8:5-13. DOI: 10.1007/BF03262564.

12. Rasmusson CG, Lundin SA. Class II restorations in six different posterior composite resins: five-year results. Swed Dent J 1995;19: 173-182.

13. Berg JH. The continuum of restorative materials in pediatric dentistry-a review for the clinician. Pediatr Dent 1998;20:93-100.

14. Holland IS, Walls AW, et al. The longevity of amalgam restorations in deciduous molars. Br Dent J 1986;161:255-258. DOI: 10.1038/ sj.bdj.4805948.

15. Roberts JF, Attari N, et al. The survival of resin modified glass ionomer and stainless steel crown restorations in primary molars, placed in a specialist paediatric dental practice. Br Dent J 2005;198:427-431. DOI: $10.1038 /$ sj.bdj.4812197.

16. Einwag J, Dunninger P. Stainless steel crown versus multisurface amalgam restorations: an 8-year longitudinal clinical study. Quintessence Int 1996;27:321-323.

17. Wong FS, Day SJ. An investigation of factors influencing the longevity of restorations in primary molars. J Int Assoc Dent Child 1990;20:11-16.

18. Seale NS. The use of stainless steel crowns. Pediatr Dent 2002;24: 501-505.

19. Blinkhorn A, Zadeh-Kabir R. Dental care of a child in pain - a comparison of treatment planning options offered by GDPs in California and the North-west of England. Int J Paediatr Dent 2003;13:165-171. DOI: 10.1046/j.1365-263X.2003.00454.x.

20. Threlfall AG, Pilkington $L$, et al. General dental practitioners' views on the use of stainless steel crowns to restore primary molars. Br Dent J 2005;199:453-455. DOI: 10.1038/sj.bdj.4812746.

21. Roshan D, Curzon ME, et al. Changes in dentists' attitudes and practice in paediatric dentistry. Eur J Paediatr Dent 2003;4:21-27.

22. Innes N, Evans $\mathrm{D}$, et al. The Hall Technique for managing carious primary molars. Dent Update 2009;36(472-474):7-8. DOI: 10.12968/ denu.2009.36.8.472. 
23. Welbury RR. The Hall Technique 10 years on: its effect and influence. Br Dent J 2017;222:421-422. DOI: 10.1038/sj.bdj.2017.262.

24. Santamaria RM, Innes NP, et al. Caries management strategies for primary molars: 1-yr randomized control trial results. J Dent Res 2014;93:1062-1069. DOI: 10.1177/0022034514550717.

25. Santamaria RM, Innes NP, et al. Acceptability of different caries management methods for primary molars in a RCT. Int J Paediatr Dent 2015;25:9-17. DOI: 10.1111/ipd.12097.

26. Innes NP, Evans DJ, et al. Sealing caries in primary molars: randomized control trial, 5-year results. J Dent Res 2011;90:1405-1410. DOI: 10.1177/0022034511422064.

27. Innes NP, Evans DJ, et al. The Hall Technique; a randomized controlled clinical trial of a novel method of managing carious primary molars in general dental practice: acceptability of the technique and outcomes at 23 months. BMC Oral Health 2007;7:18. DOI: 10.1186/1472-6831-7-18.

28. Innes NP, Ricketts $D$, et al. Preformed crowns for decayed primary molar teeth. Cochrane Database Syst Rev 2015; CD005512. DOI: 10.1002/14651858.CD005512.pub3.

29. Moher D, Liberati $A$, et al. Preferred reporting items for systematic reviews and meta-analyses: the PRISMA statement. Ann Intern Med 2009;151:264-269. DOI: 10.7326/0003-4819-151-4-200908180-00135.

30. Higgins JP, Altman DG, et al. The Cochrane Collaboration's tool for assessing risk of bias in randomised trials. BMJ 2011;343:d5928. DOI: 10.1136/bmj.d5928.

31. Zeng X, Zhang Y, et al. The methodological quality assessment tools for preclinical and clinical studies, systematic review and metaanalysis, and clinical practice guideline: a systematic review. J Evid Based Med 2015;8:2-10. DOI: 10.1111/jebm.12141.
32. Tonmukayakul U, Martin R, et al. Protocol for the Hall Technique study: A trial to measure clinical effectiveness and cost-effectiveness of stainless steel crowns for dental caries restoration in primary molars in young children. Contemp Clin Trials 2015;44:36-41. DOI: 10.1016/ j.cct.2015.07.005.

33. Hesse D, de Araujo MP, et al. Atraumatic Restorative Treatment compared to the Hall Technique for occluso-proximal cavities in primary molars: study protocol for a randomized controlled trial. Trials 2016;17:169. DOI: 10.1186/s13063-016-1270-z.

34. Amourette $L$, Vaysse $F$, et al. Faculté de chirurgie d. Approches thérapeutiques non conventionnelles de la carie en pédodontie. France: Universite Toulouse III; 2013.

35. Innes NP, Stirrups DR, et al. A novel technique using preformed metal crowns for managing carious primary molars in general practice - a retrospective analysis. Br Dent J 2006;200:451-454. DOI: 10.1038/ sj.bdj.4813466.

36. Ludwig $\mathrm{KH}$, Fontana $\mathrm{M}$, et al. The success of stainless steel crowns placed with the Hall technique: a retrospective study. J Am Dent Assoc 2014;145:1248-1253. DOI: 10.14219/jada.2014.89.

37. Santamaria RM, Innes NPT, et al. Alternative Caries Management Options for Primary Molars: 2.5-Year Outcomes of a Randomised Clinical Trial. Caries Res 2017;51:605-614. DOI: 10.1159/ 000477855.

38. Boyd DH, Page LF, et al. The Hall Technique and conventional restorative treatment in New Zealand children's primary oral health care - clinical outcomes at two years. Int J Paediatr Dent 2018;28: 180-188. DOI: 10.1111/ipd.12324. 


\section{Annexure}

ARHQ Methodology Checklist for Cross-sectional Study

Website: http://www.ncbi.nlm.nih.gov/books/NBK35156/

The Agency for Healthcare Research and Quality
1. Define the source of information (survey, record review)

2. List inclusion and exclusion criteria for exposed and unexposed subjects (cases and controls) or refer to previous publications

3. Indicate time period used for identifying patients

4. Indicate whether or not subjects were consecutive if not population-based

5. Indicate if evaluators of subjective components of study were masked to other aspects of the status of the participants

6. Describe any assessments undertaken for quality assurance purposes (e.g., test/retest of primary outcome measurements)

7. Explain any patient exclusions from analysis

8. Describe how confounding was assessed and/or controlled

9. If applicable, explain how missing data were handled in the analysis

10. Summarize patient response rates and completeness of data collection

11. Clarify what follow-up, if any, was expected and the percentage of patients for which incomplete data or follow-up was obtained 